\title{
DO CRACKS MELT THEIR WAY THROUGH SOLIDS?*
}

\author{
P. R. Okamoto and N. Q. Lam \\ Argonne National Laboratory \\ Materials Science Division \\ 9700 S. Cass Ave. \\ Argonne, IL 60439
}

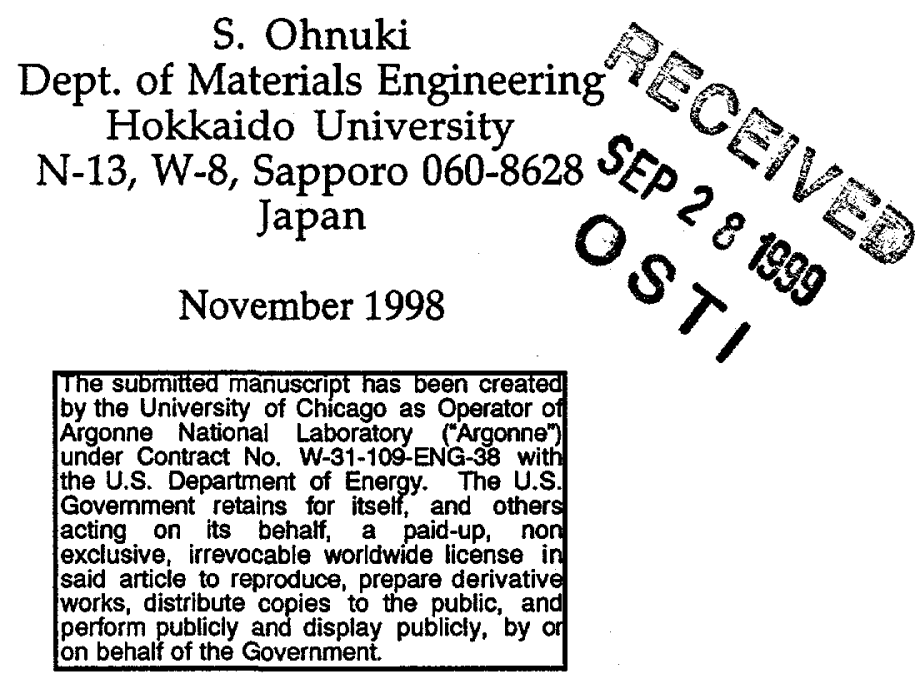

Invited manuscript to be presented at the International Symposium on High Voltage Electron Microscopy for the $21^{\text {st }}$ Century and Its Application to Frontier Materials Study, Sapporo, Japan, October 15-17, 1998.

*Work supported by the U.S. Department of Energy, Basic Energy Sciences-Materials Sciences, under contract No. W-31-109-Eng-38. 


\section{DISCLAIMER}

This report was prepared as an account of work sponsored by an agency of the United States Government. Neither the United States Government nor any agency thereof, nor any of their employees, make any warranty, express or implied, or assumes any legal liability or responsibility for the accuracy, completeness, or usefulness of any information, apparatus, product, or process disclosed, or represents that its use would not infringe privately owned rights. Reference herein to any specific commercial product, process, or service by trade name, trademark, manufacturer, or otherwise does not necessarily constitute or imply its endorsement, recommendation, or favoring by the United States Government or any agency thereof. The views and opinions of authors expressed herein do not necessarily state or reflect those of the United States Government or any agency thereof. 


\section{DISCLAIMER}

Portions of this document may be illegible in electronic image products. Images are produced from the best available original document. 


\title{
DO CRACKS MELT THEIR WAY THROUGH SOLIDS?
}

\author{
Paul R. Okamoto \\ Materials Science Division \\ Argonne National Laboratory, \\ Argonne, IL 60439 USA \\ phone: $630-252-5052$ \\ FAX: $630-252-4289$ \\ e-mail: okamoto@anl.gov \\ Nghi Q. Lam \\ Materials Science Division \\ Argonne National Laboratory, \\ Argonne, IL 60439 USA \\ phone: $630-252-4953$ \\ FAX: $630-252-4289$ \\ e-mail: nlam@anl.gov \\ Somei Ohnuki \\ Department of Materials Engineering \\ Hokkaido University \\ N-13, W-8, Sapporo 060-8628, Japan \\ phone: $81+11-706-6769$ \\ FAX: 81+11-706-6772 \\ e-mail: ohnuki@loam-ms.eng.hokudai.ac.jp
}

Send all correspondence to Paul R. Okamoto

Keywords: melting, amorphization, disorder, fracture, intermtallic compounds, NiTi

Total Pages: 15

Total Figures: 7 


\title{
Do Cracks Melt Their Way Through Solids?
}

\author{
P. R. Okamoto and N. Q. Lam \\ Materials Science Division, Argonne National Laboratory, Argonne, IL 60439, USA \\ S. Ohnuki, \\ Department of Materials and Engineering, Hokkaido University, Sapporo, Japan
}

\begin{abstract}
Real-time, in situ fracture studies in the high-voltage electron microscope (HVEM) show that microscopically thin regions of amorphous NiTi form ahead of moving crack tips in the B2-NiTi intermetallic compound during tensile straining at temperatures equal to or below $600 \mathrm{~K}$. The upper cutoff temperature of $600 \mathrm{~K}$ for this stress-induced melting (or amorphization) is identical to the upper cutoff temperatures reported in the literature for both heavy-ion-induced amorphization of the intermetallic NiTi and ion-beam-mixing-induced amorphization of $\mathrm{Ni}$ and $\mathrm{Ti}$ multilayers. These results, together with the fact that the higher crystallization temperatures $(-800 \mathrm{~K})$ of unrelaxed amorphous NiTi alloys obtained by rapid quenching can also be reduced to, but not lower than $600 \mathrm{~K}$, by heavy-ion irradiation, strongly suggest that structural relaxation processes enhanced or induced by dynamic atomic disordering allow the formation of a unique, fully-relaxed glassy state which is characterized by a unique isothermal crystallization temperature. We believe that this unique temperature is the Kauzmann glass-transition temperature, corresponding to the ideal glass having the same entropy as the crystalline state. As the glassy state with the lowest global free energy, the preferential formation of this ideal glass by disorder-induced amorphization processes can be understood as the most energetically-favored, kinetically-constrained melting response of crystalline materials driven far from equilibrium at low temperatures.
\end{abstract}

\section{INTRODUCTION}

Predicting and explaining the fracture behavior of intermetallic compounds are very important technological problems since low temperature brittleness severely limits the practical applications of many of these compounds. Despite a long and continuing effort to understand the brittle behavior, there is no generally-accepted explanation why some intermetallics are more susceptible to brittle fracture than others. The criterion for brittle fracture is usually described in terms of Griffith's thermodynamic energy criterion [1], which stipulates that a crack becomes unstable and begins to propagate when the mechanical energy release rate (which actually has units of surface 
energy), $G_{\text {cleav }}$, exceeds twice the surface energy, $\gamma_{s}$, of the two newly-created fracture surfaces, i.e. $G_{\text {cleav }}>2 \gamma_{s}$. Elasticity theory [2] can be used to calculate the local energy release rate at the crack tip from stress intensity factors specifying the applied loading conditions. However, although the local stress intensification in the immediate vicinity of the crack tip is known to be the primary driving force for fracture, the actual mechanism by which the strain energy is dissipated during crack propagation is not well understood. Since brittle fracture involves breaking of atomic bonds at the crack tip, an intriguing question is whether the bond-breaking process can be regarded as local melting of the crack tip. In the present paper, recent observations of stressinduced amorphization at moving crack tips in the intermetallic compounds NiTi are discussed, which strongly supports the idea that cleavage cracks melt their way through solids.

\section{THEORETICAL BACKGROUND}

A simple model of cleavage fracture based on melting concepts was proposed in 1971 by Caglioti et al. [3] who hypothesized that melting of the crack tip occurs when adjoining atoms pairs across the cleavage plane are displaced a critical amount beyond their equilibrium positions. The critical atomic displacement was assumed to be the mean-square thermal displacement that satisfies the Lindemann criterion for melting, which in the harmonic approximation for a Debye solid is

$$
<\mu_{\mathrm{cin}}^{2}>=\frac{9 \hbar^{2} \mathrm{~T}_{\mathrm{m}}}{\mathrm{mk} \theta^{2}}
$$

where $T_{m}$ and $\theta$ are, respectively, the thermodynamic melting and Debye temperatures of the perfect crystal, $\mathrm{m}$ is the atomic mass, $\mathrm{k}$ is the Boltzmann constant, and $\hbar$ is Plank's constant divided by $2 \pi$. The Lindemann melting criterion for fracture leads to a very simple estimate for the surface energy [3]

$$
\gamma_{\mathrm{S}}=\frac{\mathrm{m} \omega^{2}}{4}<\mu_{\mathrm{cri}}^{2}>\mathrm{n}+\mathrm{k}\left(\mathrm{T}_{\mathrm{B}}-\mathrm{T}_{\mathrm{m}}\right)
$$

In the first term, which is the potential energy associated with the critical displacement of adjoining atoms pairs, $\mathrm{m} \omega^{2} / 4$ is the nearest-neighbor force constant, $\omega$ is the frequency of the lattice vibration and $\mathrm{n}$ is the number density of atoms per unit area in the plane of interest. The second term is the energy required to separate the two fractured surfaces to a distance where they no longer influence each other and is taken as the energy difference between the boiling temperature of the molten liquid, $\mathrm{T}_{\mathrm{B}}$, and the melting temperature of the solid. Despite the simplicity of the model, the cal- 
culated surface energies of a number of elements are in suprisingly good agreement with experimental values, particularly in predicting the correct cleavage plane for zinc and tungsten [3].

It follows from Eq. (1) that the melting temperature of the perfect crystal will be given by

$$
T_{m}=\frac{m k \theta^{2}}{9 \hbar^{2}}<\mu_{c i}^{2}>
$$

However, Okamoto et al.[4] pointed out that the melting temperature of highly-strained regions of crystals, where atoms suffer large static displacements from their equilibrium positions, will not be the same as that of a defect-free crystal described by Eq.(3), which considers only the effects of dynamic (thermal) displacements. Their generalized version of the Lindemann melting criterion assumes that melting of a defective crystal occurs when the sum of the static and dynamic meansquare displacements reaches a critical value identical to that for melting of the perfect crystal, i.e.

$$
\frac{9 \hbar^{2} \mathrm{~T}_{\mathrm{m}}^{\mathrm{d}}}{\mathrm{mk} \theta_{\mathrm{d}}^{2}}+<\mu_{\mathrm{sta}}^{2}>=<\mu_{\mathrm{cri}}^{2}>
$$

If this hypothesis is correct, the bulk melting temperature of a defective crystal, or the local melting temperature of the highly strained crack tip region of a crystal, will be given by

$$
\mathrm{T}_{\mathrm{m}}^{\mathrm{d}}=\frac{\mathrm{mk} \theta_{\mathrm{d}}^{2}}{9 \hbar^{2}}<\mu_{\mathrm{cri}}^{2}>
$$

which is identical in form as Eq. (3), but with a disorder-dependent Debye temperature given by

$$
\theta_{d}^{2}=\theta^{2}\left[1-\frac{\left\langle\mu_{\mathrm{sta}}^{2}\right\rangle}{\left\langle\mu_{\mathrm{cri}}^{2}\right\rangle}\right] .
$$

Equation (5) predicts that the melting temperature of a defective crystal will decrease linearly with increasing degree of disorder, measured by $\left\langle\mu_{\mathrm{sta}}^{2}\right\rangle$, reflecting the effect of disorder on the Debye 
temperature described by Eq. (6). Since $\theta^{2}$ of an isotropic elastic solid scales linearly with the average shear modulus $G$, the linear decrease in melting temperature with increasing disorder will be directly observable as a disorder-induced elastic softening of the average shear modulus having the same functional dependence on $\left\langle\mu_{\text {sta }}^{2}>\right.$ as Eq. (6). Hence, a direct corollary of the generalized Lindemann melting criterion is that the reduced quantities $T_{m}^{d} / T_{m}, \theta_{d}^{2} / \theta^{2}$, and $G_{d} / G$, all must be equal and have the same functional dependence on $\left\langle\mu_{\mathrm{sta}}^{2}\right\rangle$, i.e.

$$
\frac{\mathrm{T}_{\mathrm{m}}^{\mathrm{d}}}{\mathrm{T}_{\mathrm{m}}}=\frac{\theta_{\mathrm{d}}^{2}}{\theta^{2}}=\frac{\mathrm{G}_{\mathrm{d}}}{\mathrm{G}}=\left[1-\frac{\left\langle\mu_{\mathrm{sig}}^{2}\right\rangle}{\left\langle\mu_{\mathrm{cri}}^{2}\right\rangle}\right]
$$

The lowering of the melting temperature reflecting the elastic softening induced by a defect structure, as described by Eq. (7) is a very general effect since it depends on the magnitude, but not on the physical origin of $\left\langle\mu_{\mathrm{sta}}^{2}\right\rangle$. Hence, $\left\langle\mu_{\mathrm{sta}}^{2}\right\rangle$ can be a measure of the concentrations of point defects, misfitting solute atoms, anti-site defects, or of the dislocation density or any other type of intrinsic or extrinsic defects, including inhomogeneous defect structures like grain boundaries (see ref. [4] ),. For example, if $\mathrm{C}$ is the atomic fraction of point defects or misfitting solutes in a crystal, the mean-square static displacement associated with these defects will be of the form $<\mu_{\text {sta }}^{2}>\alpha \mathrm{C}(1-\mathrm{C})$, and hence will, be directly proportional to their concentrations for $\mathrm{C}<<1$; for anti-site defects in an ordered alloy or intermetallic compound, $<\mu_{\mathrm{sta}}^{2}>\alpha\left(1-\mathrm{S}^{2}\right)$, where $\mathrm{S}$ is the Bragg-Williams long range order parameter; for a dislocation structure of density $\rho,<\mu_{\mathrm{sta}}^{2}>\alpha \rho^{2}$; and for grain boundaries of average diameter $\mathrm{D},<\mu_{\mathrm{sta}}^{2}>\alpha \mathrm{D}^{1} . \mathrm{T}_{\mathrm{m}}^{\mathrm{d}}$ in Eq. (5) or (7), therefore represents a generalized polymorphous melting curve for defective crystals, which, for example, can be used to obtain conventional $\mathrm{T}_{\mathrm{o}}$-curves on binary phase diagrams by expressing $<\mu_{\mathrm{sta}}^{2}>$, $\theta$ or $\mathrm{G}$ in these equations as a functions of alloy composition $[4,5]$. The Lindemann scaling relationships in Eq. (7) are of special importance for damage accumulation processes, such as irradiation or ball milling, since the effect of damage on the melting temperature will not be directly measurable due to annealing of the damage structure during heating. However, at very low temperatures, $\theta_{d}$ or $G_{d}$ can be readily measured as a functions of defect concentration [6], or degree of chemical long-range order [7], and hence, the corresponding polymorphous melting curve for the damage crystal can be determined using Eq. (7). 
An important prediction of the generalized Lindemann melting criterion is that any crystal can be forced to melt below the thermodynamic melting point of the defect-free crystal by introducing a sufficient amount of static disorder. That applied tensile stresses can drive this disorderinduced melting at crack tips, due to the local stress intensification, is supported by recent Monte Carlo (MC) simulations of crack formation [8]. Figure 1a shows a stress-strain curve calculated for a model fcc system under uniaxial tension at $T=0.35 \mathrm{~T}_{\mathrm{m}}$ using a Finnis-Sinclair type potential fitted to platinum. The stress-induced melting event is seen as a sudden drop in the stress level at a strain of $\varepsilon_{\mathrm{cn}} \approx 0.13$. The sequence of atomic configurations in Fig. $1 \mathrm{~b}$ show that, as the crystal is stretched, the displacement of atoms from their equilibrium increases until a temperature-dependent critical strain is reached ( $\varepsilon_{\mathrm{cri}} \approx 0.13$ for $\mathrm{T}=0.35 \mathrm{~T}_{\mathrm{m}}$ ) where thermal fluctuations induces local melting. The sudden drop in the stress level reflects the fact that the supercooled liquid cannot sustain a tensile load, and hence, rapidly resolidifies expitaxially, leaving a cavity as the only evidence that melting has occurred.

On the basis of molecular dynamic simulations, Wolf and co-workers [9] have suggested that expansion-driven melting can be triggered by a mechanical instability corresponding to the vanishing of a shear elastic constant. However, free energy calculations by Lynden-Bell [8], show that the stress-induced melting at ambient temperatures occur at a critical strain (or stress), where the free energy of the stretched crystal becomes equal to free energy of the supercooled liquid at the ambient temperature. Hence, the MC simulation shows that melting occurs at a critical combination of temperature and applied stress where the thermodynamic melting temperature for that stress level becomes equal to the local ambient temperature at the crack tip. The lower the ambient temperature, the higher the applied stress level required for melting. This is illustrated by the $M C$ results in Fig. 2, which show that the melting temperature of the stretched crystal $T_{m}(\sigma)$ decreases linearly with increasing applied stress. It also shows that the effect of stress in lowering the melting temperature is identical to that of $\left\langle\mu_{\mathrm{sta}}^{2}\right\rangle$ in the generalized Lindemann melting criterion, which is not surprising since the atomic configurations in Fig. 1b show that $<\mu_{\text {sta }}^{2}>$ increases with applied stress.

All of these stress effects can be easily understood by examining Fig. 3, which displays the temperature dependence of the Gibbs free energy of the unstressed crystal, of the liquid, and of two stressed crystalline states indicated by the dotted curves. Also shown are the free energy curves for the ideal glass and two unrelaxed glassy states 1 and 2 , associated with cooling rates 
such that $T_{g 1}>T_{g 2}>T_{K}$, where $T_{K}$ is the Kauzmann glass transition temperature [10] corresponding to the ideal glass, i.e. the glass having the same entropy as the perfect crystal. $T_{m}(\sigma)$ and $T_{m}(\sigma=0)$ are the thermodynamic melting temperatures of the stressed and unstressed crystals, respectively, as defined by the intersection of their free energy curves with that of the liquid. Figure 3 shows that as the free energy of the stressed-crystal increases, its melting temperature decreases and approaches the ideal glass transition temperature, $T_{K}$, as the free energy of the stretched crystal approaches that of the ideal glass. Hence, the thermodynamic criterion for stressinduced glass formation becomes $T_{m}(\sigma)=T_{K}$. It follows from the Lindemann scaling relationships in Eq. (7) that this thermodynamic criterion can be expressed as an elastic softening criterion, i.e., $G_{d}=G_{K}$ or $\theta_{d}=\theta_{K}$, where the subscript $K$ denotes the ideal glassy phase. Figure 3 also shows that, at this critical point. the free energy curve of the stretched crystal becomes tangent to that of the ideal glass, implying that the melting transition, becomes a continuous or second order crystal-to-glass transition that results in the formation of that glass having the lowest global free energy. Two very important consequences follow from this thermodynamic picture. The first is that the formation of the ideal glass will always be favored by solid-state disordering processes over the formation of any unrelaxed glassy state. The second is that $T_{K}$ represents an absolute upper-limiting cutoff temperature for all disorder-driven crystal-to-glass transformations. This conclusion follows from the fact there is no thermodynamic driving force for amorphization for any disordered or stressed crystalline state that has a melting temperature higher than $T_{K}$. In the following section recent experimental observations [11] supporting this simple thermodynamic picture of disorder-driven melting at crack tips are examined.

\section{EXPERIMENTAL OBSERVATIONS AND DISCUSSION}

Although MC simulation predicts that brittle fracture via local melting of crack tips is thermodynamically possible, in practice there are kinetic reasons why it may be difficult, if not impossible to observe in simple fcc metals. First, as shown by the simulations, the melting process for fcc metals is a short-lived transient phenomenon where rapid epitaxial resolidification of the supercooled liquid may leave no evidence that melting has occurred. And second, other energy dissipative processes, such as dislocation emission from crack tips, may occur first, i.e., at stress levels lower than that required for melting. However, these effects can be circumvented for intermetallic compounds, since many can be amorphized during irradiation with energetic particles at very low temperatures where most materials, with the possible exception of fec metals, fail in a brittle manner. Since the radiation-induced crystal-to-glass transformation is simply disorderinduced melting of a critically-damaged crystal to a frozen supercooled liquid, i.e. to a glass, it 
should be possible to observe stress-induced melting at very low temperatures as amorphization of the crack tip in a stressed intermetallic compound. Furthermore, as discussed in section 2 , the resulting glass should be the same ideal glass produced by other types of disordering processes such as ion implantation or ion-beam mixing. Earlier, we reported direct HVEM observations of stress-induced amorphization at moving crack tips during straining of the intermetallic compound NiTi [11]. The temperature dependence of stress-induced amorphization and the isothermal crystallization behavior of the resulting glass were compared with those associated with irradiationinduced amorphization of crystalline $\mathrm{NiTi}$ [12] and with ion-beam mixing of $\mathrm{Ni}$ and $\mathrm{Ti}$ multilayers [13]. The results confirmed our hypothesis that, like the other solid-state amorphizing transformations, stress-induced melting of crack tips below $T_{K}$ leads to the formation of a unique ideal glassy state characterized by an isothermal crystallization temperature of $600 \mathrm{~K}$.

In situ fracture observations in NiTi were carried out with a Gatan side-entry heatingstraining stage in the HVEM at the Argonne National Laboratory HVEM-Tandem accelerator facilities. The straining stage is capable of achieving temperatures between $300-825 \mathrm{~K}$ and a maximum elongation of $2 \mathrm{~mm}$. Miniature NiTi tensile specimens were prepared from bulk alloys with Ti concentrations between $47-49$ at. $\%$ Ti. The bulk alloys were rolled down to $0.1 \mathrm{~mm}$ thick sheets which were subsequently cut into $3.0 \times 6.5 \mathrm{~mm}$ strips. Tensile specimens for the Gatan straining stage were prepared from these strips by boring 1-mm diameter holes in the two ends for mounting on the straining stage. A gauge length was created by electro-etching two slits along the length of the specimen. All specimens were given a high-vacuum, pre-strain anneal at $900^{\circ} \mathrm{C}$ for 20 minutes to remove any mechanical damage introduced during preparation. The annealing treatment resulted in polycrystalline B2-NiTi with a typical grain size of $10 \mu \mathrm{m}$. Prior to mounting, the central area in the gauge length was electropolished to electron transparency, using a single-jet electropolisher with custom polishing holder.

In situ studies of stress-induced amorphization at crack tips on NiTi tensile specimens were carried out between 300 - 750K. Microscopy observations were carried primarily in the dark-field (DF) mode since the thin amorphous regions which form along crack edges and tips are extremely difficult to see in bright field images. Figure 4 shows a DF image of a typical crack formed during straining at $300 \mathrm{~K}$ which is well below the range of kinetic glass transition temperatures 750 $850 \mathrm{~K}$ reported for amorphous NiTi produced by rapid quenching techniques [14]. Selected area diffraction (SAD) patterns taken from areas away from the crack tip region indicate a crystalline structure while the $\mathrm{SAD}$ pattern from the crack tip region exhibits diffuse intensity halos, indicating that an amorphous phase forms ahead of the crack tip. DF images taken with part of the first 
diffuse intensity halo show that the amorphous phase is also present along the edges of the crack. This indicates that, at $300 \mathrm{~K}$, the amorphous phase which forms at the crack tip remains amorphous as the crack propagates into the crystal.

The temperature dependence of stress-induced amorphization is summarized in Fig. 5, which shows the average width of the amorphous layer along the crack edges as a function of temperature measured for several NiTi specimens having either the B2 or martensitic structure prior to straining. These results show that stress-induced amorphization at crack tips only occurs for temperatures below about $600 \mathrm{~K}$. As shown in Fig. 6, this cutoff temperature can also be determined in a different way, by plotting the percentage of specimens ( 12 were examined) which exhibit either complete or partial stress-induced amorphization as a function of straining temperature. Again, one sees a relatively sharp upper cutoff temperature at about $600 \mathrm{~K}$, which is also the upperlimiting cutoff temperature $\mathrm{T}_{\mathrm{C}-\mathrm{A}}^{\max }$ for both radiation-induced amorphization of crystalline NiTi by heavy-ions [12], and amorphous phase formation by ion-beam mixing of $\mathrm{Ni}$ and $\mathrm{Ti}$ multilayer specimens [13]. The thermodynamic significance of $600 \mathrm{~K}$ as the upper limiting cutoff temperature for all three types of disorder-induced, glass formation processes is that the crystallization temperature of unrelaxed NiTi glasses produced by rapid quenching techniques is generally much higher, typically around 800K [14]. However, as shown by Moine and Jaouen [12], the crystallization temperature for the unrelaxed glasses can be lowered to $600 \mathrm{~K}$, but no lower, by heavy-ion bombardment. When taken togeher, these observation strongly suggests that structural relaxation processes enhanced or induced by dynamic atomic disordering events allows the formation of a unique, fully-relaxed NiTi glass which is characterized by an isothermal crystallization temperature of $600 \mathrm{~K}$.

There are several reasons why we believe that this temperature of $600 \mathrm{~K}$ is the Kauzmann glass transition temperature, $T_{K}$, of the ideal NiTi glassy state having the same entropy as the crystalline state. First of all, as a cutoff temperature for amorphization, which is independent of the type of disordering process used, it satisfies one of the characteristic features of $T_{K}$, namely the absence of a thermodynamic driving force for amorphization above $600 \mathrm{~K}$. Secondly, the fact that the crystallization temperature of initially unrelaxed NiTi glasses can be relaxed to, but not lower than $600 \mathrm{~K}$, is also consistent with the concept of fully-relaxed, unique glassy state. A third reason is that the cutoff temperature of $600 \mathrm{~K}$ coincides with the temperature where dramatic changes in elastic moduli and density are observed during heating of initially unrelaxed NiTi glasses. For example, Okamoto et al [4] noted that the isochronal resistivity measurements by Balanzat et al. [15] show that the equilibrium state of chemical short range order (CSRO) in initially unrelaxed NiTi glasses is also attained at about $600 \mathrm{~K}$. This attainment of equilibrium CSRO 
occurs just prior to the onset of topological short range order (TSRO) which is generally attributed to the annealing of excess "free volume". The significance of this observation is that differentialscanning-calorimetric studies [16] show that the relaxation process associated the gradual increase in CSRO involves heat absorption, whereas those related to increasing TSRO involve heat release. The fact that $600 \mathrm{~K}$ coincides with temperature at which the transition from CSRO to TSRO occurs, shows that the crystallization event occurring at $600 \mathrm{~K}$ is unique, involving neither heat release nor heat absorption, i.e. no entropy change. This is a defining signature of the ideal glass transition since no heat release implies no entropy difference between the ideal glassy state and its crystalline counterpart. Hence, the ideal glass may simply represent the equilibrium CSRO state for a glass and the Kauzmann ideal glass transition at $T_{K}$, the transition from equilibrium CSRO state to TSRO.

The ideal glass-transition temperature can also be determined by plotting the kinetic glass transition $T_{g}$ and kinetic crystallization temperatures $T_{X}$ as a function of heating rate. As shown by Busch et al. [17] for amorphous $\mathrm{Zr}_{41.2} \mathrm{Ti}_{13.8} \mathrm{Cu}_{12.5} \mathrm{Ni}_{10.0} \mathrm{Be}_{22.5}$, and by Okamoto et al. [4] for amorphous $\mathrm{CuTi}$, the two curves, $\mathrm{T}_{\mathrm{g}}$ and $\mathrm{T}_{\mathrm{x}}$ intersect at a point defining a critical heating rate and a critical temperature where $T_{g}=T_{x}$. In the case of CuTi, the critical temperature of $565 \mathrm{~K}$, defined by the intersection point coincides not only with the absolute upper-limiting cutofftemperature for radiation-induced amorphization reported by Koike et al. [17], but also with the temperature at which unrelaxed CuTi glasses attain the equilibrium state of CSRO during heating

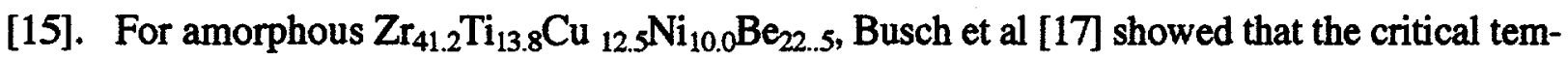
perature coincides with the ideal glass transition temperature, $T_{K}=562 \mathrm{~K}$, determined from specific heat measurements of the equilibrium supercooled liquid and crystalline states. The corresponding critical heating rates are $1.67 \times 10^{-5} \mathrm{~K} / \mathrm{min}$ for amorphous $\mathrm{Zr}_{41.2} \mathrm{Ti}_{13.8} \mathrm{Cu}_{12.5} \mathrm{Ni}_{10.0} \mathrm{Be}_{22.5}$ and $6.6 \times 10^{-4} \mathrm{~K} / \mathrm{min}$. for amorphous $\mathrm{CuTi}$, which corresponds to $1 \mathrm{~K}$ every 41 days for $\mathrm{Zr}_{41.2} \mathrm{Ti}_{13.8} \mathrm{Cu}_{12.5} \mathrm{Ni}_{10.0} \mathrm{Be}_{22.5}$ and $1 \mathrm{~K}$ every 25.3 hours for CuTi. Since $\mathrm{T}_{K}=\mathrm{T}_{\mathrm{x}}$ for these essentially zero heating rates, the ideal glass should crystallize when annealed isothermally at $T_{K}$, even though it is typically several hundreds of degrees lower than the kinetic crystallization temperatures of unrelaxed glasses. This hypothesis was tested by annealing the "ideal" glass produced at the crack tip of a NiTi specimen fractured at $300 \mathrm{~K}$ for 15 minutes at various temperatures between 300 and 923K. No change in the structure of the amorphous NiTi occurs until 593K where the first hint of crystallization is observed, thus confirming the hypothesis. This is illustrated in Fig. 7, which shows DF images and the corresponding SAD patterns after annealing at various temperatures. At $523 \mathrm{~K}$, the SAD pattern shows that the crack tip is still completely amor- 
various temperatures. At $523 \mathrm{~K}$, the SAD pattern shows that the crack tip is still completely amorphous. After annealing for 15 minutes at $593 \mathrm{~K}$, which is just below $T_{K}$, a few small crystalline grains are seen in DF images, although the SAD pattern indicates that the crack tip and regions along the crack edges are still primarily amorphous. However, after 15 minutes at $633 \mathrm{~K}$, the SAD pattern exhibits polycrystalline rings, showing that the "ideal" NiTi glass at the crack tip and edges has almost completely crystallized.

\section{CONCLUDING REMARKS}

In situ fracture studies of crystalline NiTi have confirmed recent predictions of MC simulations that stress-induced melting (amorphization) at crack tips can occur in crystals subjected to uniaxial tensile stresses. The simulations show that tensile stress plays the same role in lowering the melting temperature of stretched crystals as that played by $\left\langle\mu_{\mathrm{sta}}^{2}>\right.$ in lowering the melting temperature of defective crystals via the generalized Lindemann melting hypothesis. Isothermal crystallization studies on amorphous NiTi produced at crack tips and edges confirm the hypothesis that stress-induced amorphization, like other disorder-induced crystal-to-glass transformations, results in the formation of unique, fully-relaxed, ideal glass having the same entropy as its crystalline counterpart. There is experimental evidence that this ideal glass may correspond to the state of equilibrium degree of CSRO, and that the Kauzmann glass transition temperature may represent the transition form equilibrium CSRO to TSRO, a transition which involves no heat release or absorption, i.e. no entropy change.

This work was supported by the U.S. Department of Energy, Basic Energy Sciences-Materials Sciences, under contract W-31-109-Eng-38. Special thanks are extended to B. Kestel for his aid with tensile specimen preparation, and to E. Ryan, S. Ockers and L. Funk of the Argonne HVEMTandem accelerator facility for their assistance in performing the in situ straining experiments 


\section{REFERENCES}

1. Griffith A A, (1920) Phil. Trans. Roy. Soc. A 221:163.

2. Sih GC and Liebowitz H (1968)Fracture, ed. Liebowitz H, Vol. 2, pp. 67 (Academic Press, New York).

3. Caglioti G, Rizzi G and Bilello J C, (1971) J. Appl. Phys, 42: 4271.

4. Okamoto P R, Lam N Q, and Rehn L E, (1998) Physics of Crystal-to-Glass

Transformations, in Solid State Physics, eds. Ehrenreich H and Spaepen F, Vol. 52

(Academic Press, New York), to be published.

5. Krill III C E, Li J, Garland M, Ettl C, Samwar K, Yelon W B, and Johnson W L, (1995) J. Mater. Res. 10: 280.

6. Rehn L E, Holder J, Granato A V, Coltman R R and Young Jr. F W (1974). Phys. Rev. B 10: 349 .

7 Okamoto P R, Rehn L E, Pearson J, Bhadra R and Grimsdtich M, (1988) J. Less-Common Met. 140: 231.

8. Lynden-Bell R M, (1995) J. Phys. Condens. Matter. 7: 4603.

9. Wolf D, Okamoto P R, Yip S, Lutsko J F and Kluge M (1990) J. Mater. Res. 5: 286.

10. Kauzmann W, (1948) Chem. Rev. 43: 219.

11. Okamoto P R, Heuer J , Lam N G, Ohnuki S., Matsukawa Y, Tozawa K and Stubbins J F, (1998) Appl. Phys. Lett.: 75, 473

12. Moine $P$ and Jaouen C, (1993) Journ. Alloys Compounds :194, 373.

13. Aaen Andersen L-U, Bottinger J, Janting J and Karpe N, (1991) Mater. Sci. Eng. A134: 1255.

14. Buschow K H J, (1984) J. Appl. Phys: 56, 304.

15. Balanzat E, Stanley J T, Mairy C and Hillairet J, (1985) Acta. Metall. 33: 785.

16. Altouinian Z, (1988) Mater. Sci. Eng. 97: 461.

17. Busch R, Kim Y J, and Johnson W L, (1995) J. Appl. Phys: 77: 4039. 


\section{FIRE CAPTIONS}

Fig. 1: (a) The stress-strain curve for model platinum at $\mathrm{T}=0.35 \mathrm{~T}_{\mathrm{m}}$ (from ref. 5). (b) Changes in the atomic configurations as a function of strain showing that the dramatic drop in stress level on the stress-strain curve in Fig. 1 corresponds to the onset of local melting.

Fig. 2: MC simulation results showing that the melting temperature of the stressed crystal decreases linearly with increasing values of the critical applied stress.

Fig. 3: Schematics of the Gibbs free energy versus temperature of the stressed and unstressed crystals.

Fig. 4: Dark-field image and selected area diffraction patterns showing amorphous phase formation in the region ahead of crack tip and along the crack edges [8].

Fig. 5: Average width of amorphous zone along crack edge as a function of temperature for B2 and martensitic NiTi [8].

Fig. 6: Percentage of NiTi specimens exhibiting either complete or partial amorphization during fracture [8].

Fig. 7. Annealing of stress-induced amorphous phase at an intergranular crack in NiTi formed during straining in the HVEM at $300 \mathrm{~K}$. The specimen was annealed for 15 minutes at each temperature. 


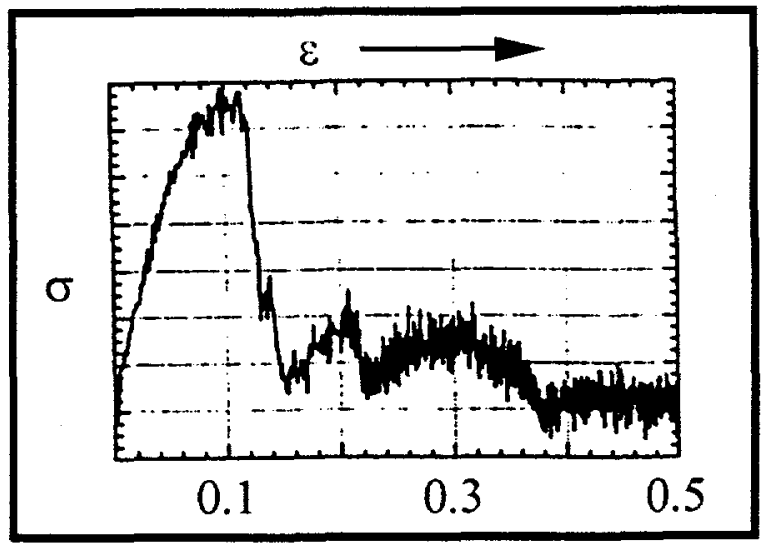

(a)

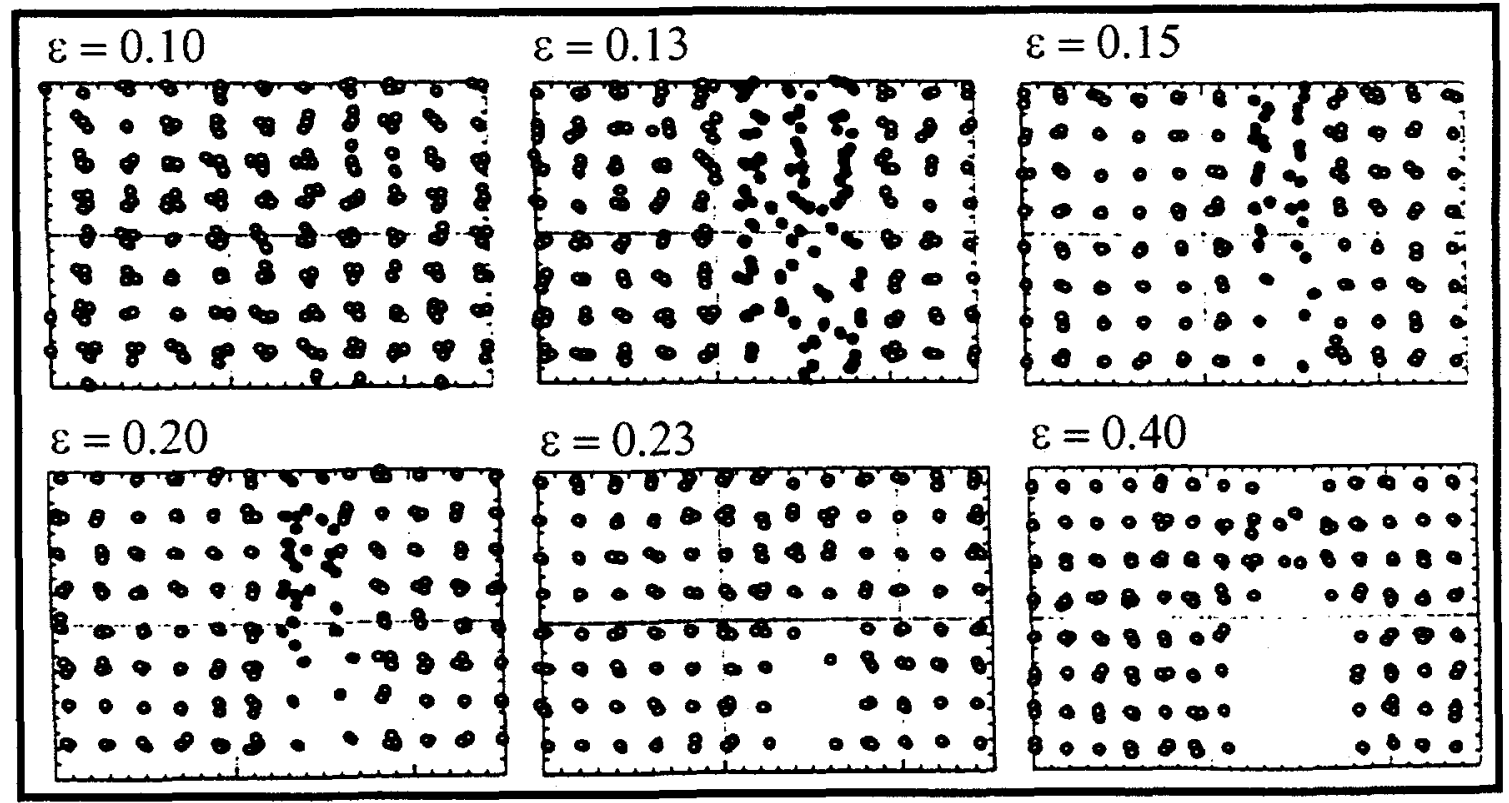

(b) 


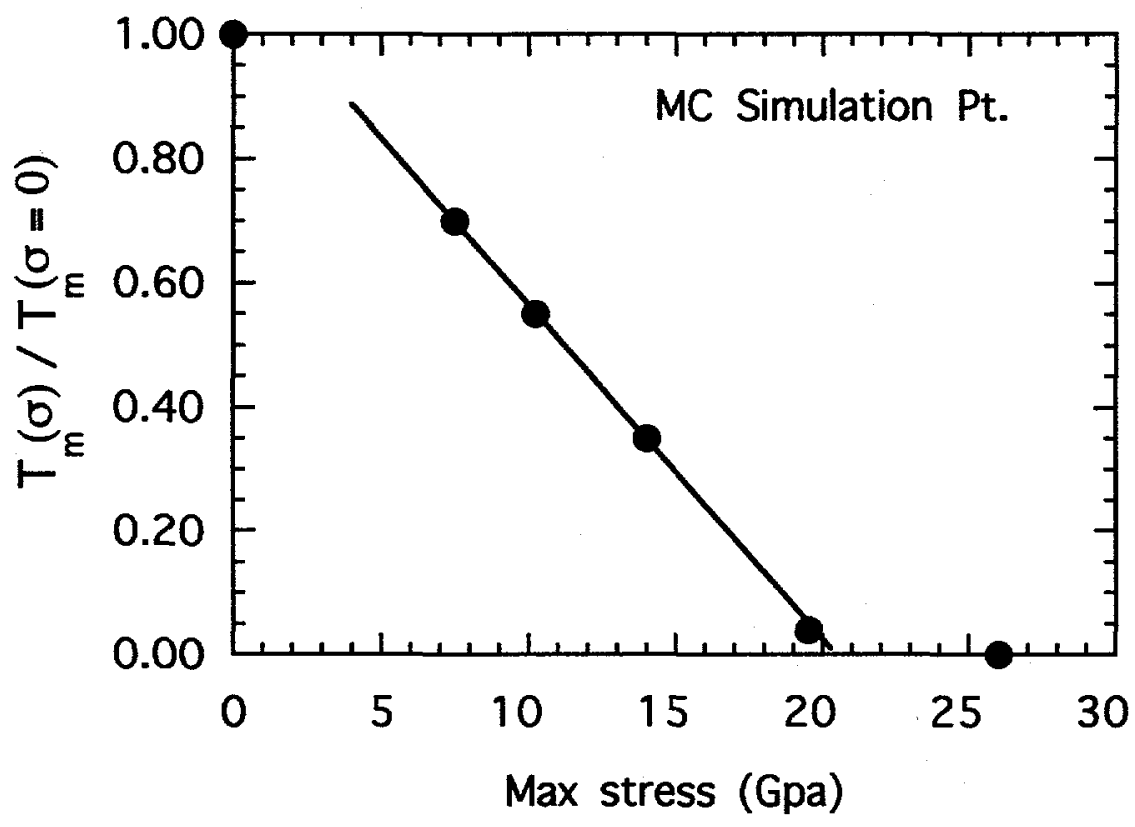




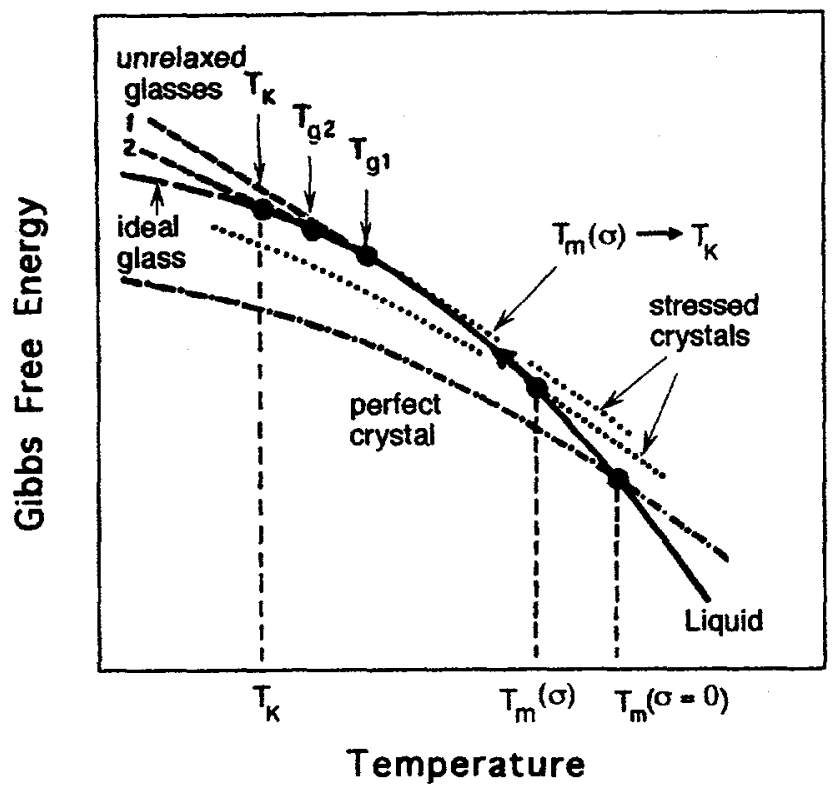




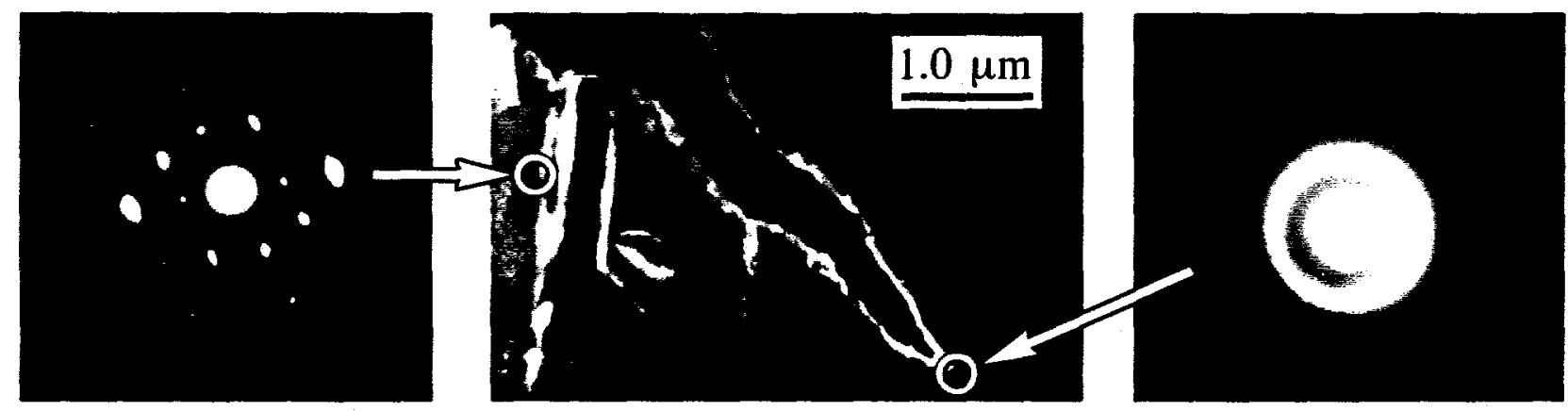

Fig. 24 


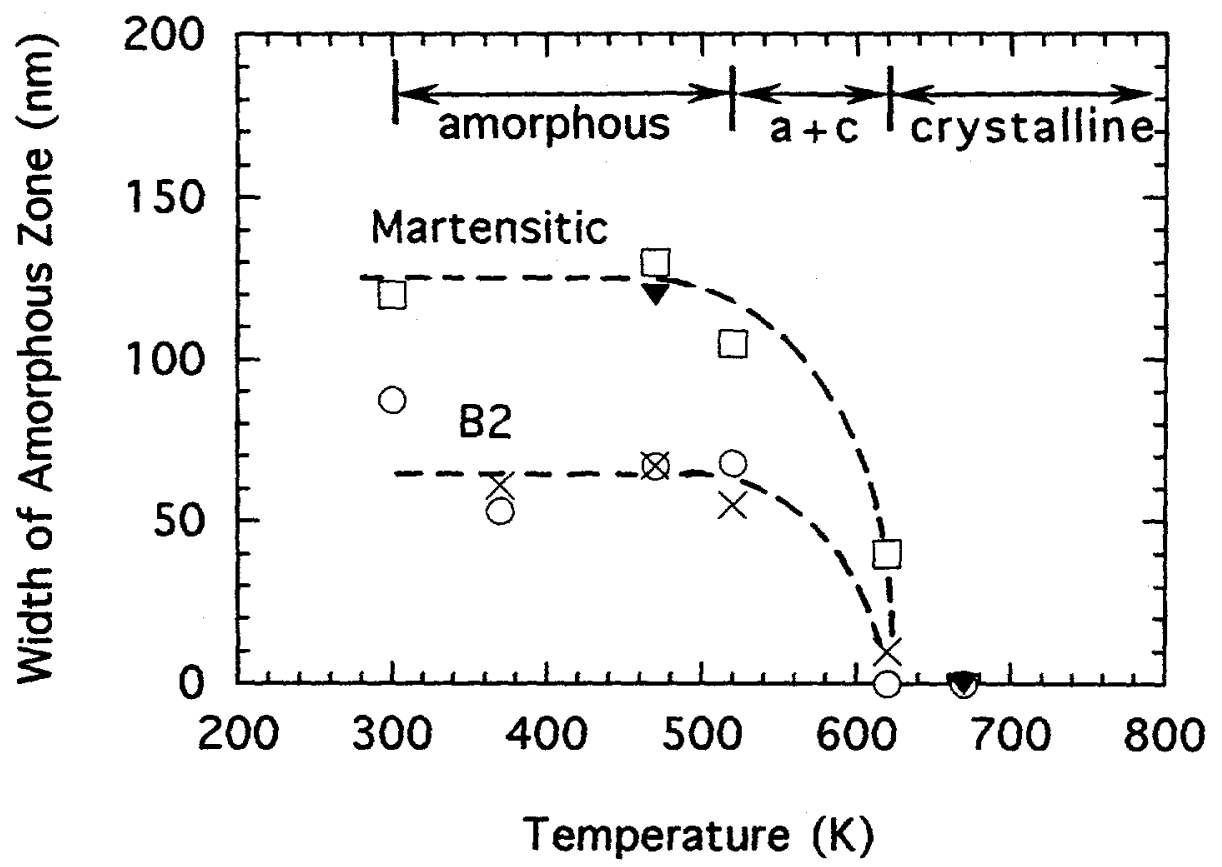




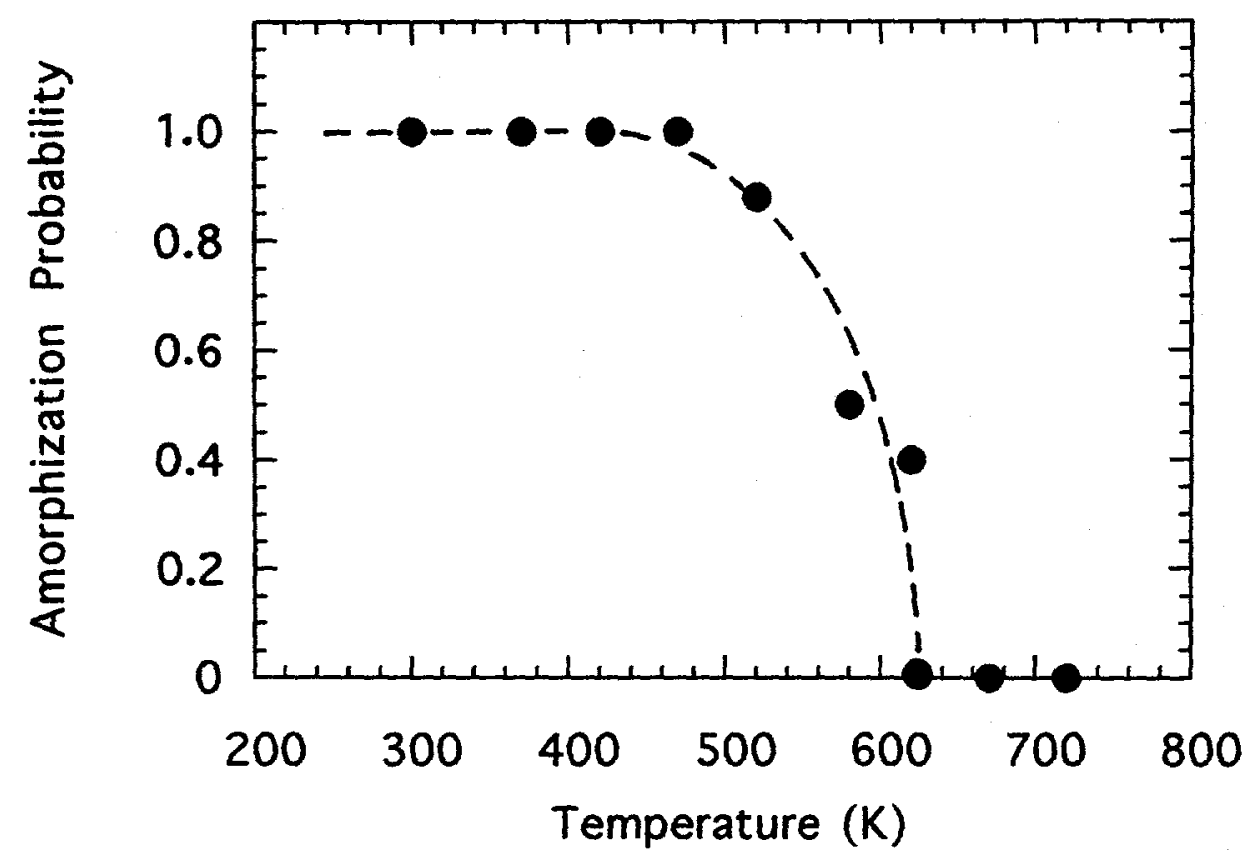




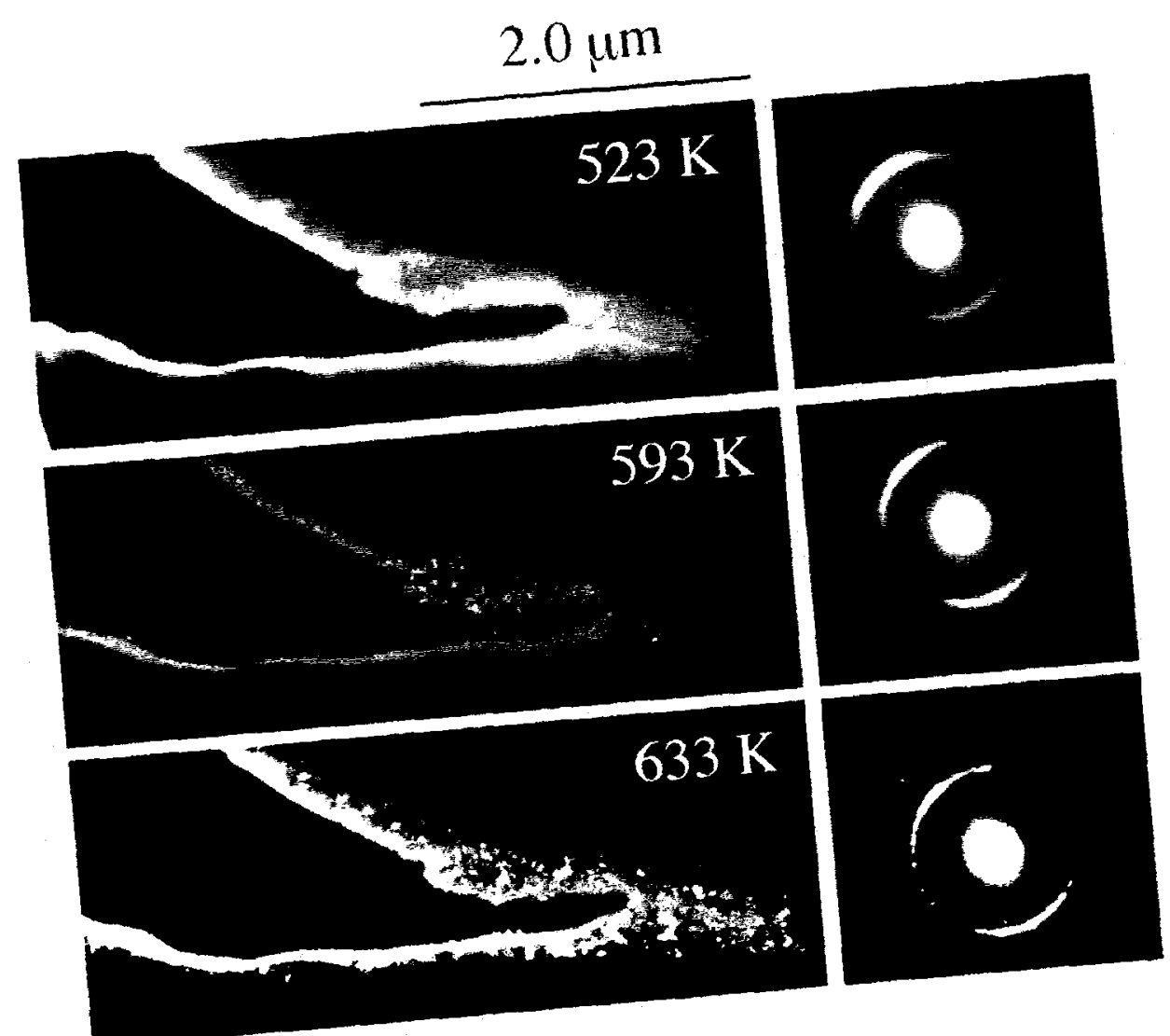

